\title{
CONSIDERazIONI SUL PASSato PROSSIMO
}

\author{
Jussara de Fatima Mainardes Ribeiro \\ Universidade Federal do Paraná
}

\begin{abstract}
RIASSUNTO
Questo lavoro pretende presentare e mettere in risalto l'importanza, l'origine, l'uso e la formazione del Passato Prossimo, tempo verbalc fondamentale alla comunicazione nella lingua italiana, da caratterizzarsi come un tempo passato, ma con riferimento al presente.
\end{abstract}

\section{INTRODUZIONE}

Ogni cultura possiede caratteristiche proprie, originate da diversi fattori. Quindi, ogni lingua umana codifica il suo universo per mezzo di vocaboli in modi diversi. Ogni lingua è un sistema a sé, differente da tutti gli altri.

La ricchezza del sistema verbale delle lingue neolatine, in confronto con il sistema nominale, è cosa troppo risaputa. Il sistema verbale è la parte piú importante della morfosintassi di tutte le lingue romanze e presenta, per conseguenza, un grande numero di variazioni.

La nozione di tempo è, probabilmente, comune a tutte le culture del mondo, però, questa nozione è espressa in maniere diverse dalle lingue del mondo. $\mathrm{E}$ il verbo fa suporre il tempo, tra altre categorie.

Nel presente lavoro tenteremo presentare un tempo di rerbo denominato Passato Prossimo, che esercita un'importante funzione nella lingua italiana per il suo immenso valore espressivo e quindi, con un uso assai costante, dovendo costituirsi in uno dei primi tempi verbali fondamentali nell'apprendimento dell'italiano.

In italiano, solo il passato ha cinque tempi: 


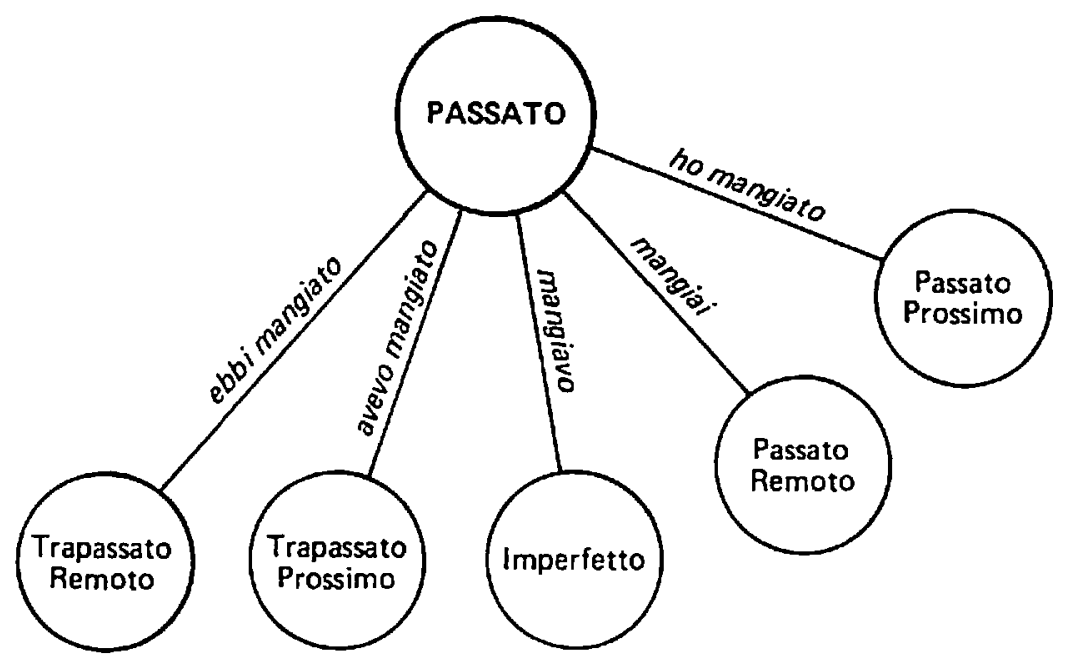

Tra questi, distacchiamo il Passato Prossimo, usato nelle descrizioni e nelle narrazioni di avvenimenti accaduti in un tempo passato, ma non del tutto trascorso.

\section{L'ORIGINE}

La nascita del passato prossimo rimonta all'epoca latina classica. Lo troviamo in Plauto: illa omnia missa habeo, omnes res relictas habeo $e$ in Cicerone satis habeo deliberatum, scriptum habeo, pecunias magnas colocatas habent.

Esprimeva in origine uno stato o un effetto duraturo: epistolam scriptam habeo "ho una lettera che è scritta". Man mano che il soggetto veniva sempre piú pensato come res. ponsabile dell'azione, o esecutore del fatto, la costruzione poté venire a caratterizzare l'attività stessa. In seguito, il trapasso a tempo del passato si consolidò ancor piú. Solo in epoca molto piu tarda la costruzione venne estesa ai verbi intransitivi: habeo dormitum "ho dormito".

Nel toscano quest'ultima fase è stata realizzata solo in parte. Mentre i verbi transitivi vengon tutti costruiti con avere, gli intransitivi vogliono in parte essere, in parte avere. ${ }^{1}$

\section{L'USO}

Il passato prossimo indica uno stato che si è realizzato nel passato con effetto continuato fino al presente:

1 RoFlFs. Qerhard. Grammatica storica dalla lingua ttallara e del suos dialettl. Torino. Elnaudi Editore. 1969. $\%$. 3. p.119. 
Tua sorella è stata sempre una ragazza studiosa.

O un'azione compiuta nel passato, ma i cui effetti hanno una qualche relazione col presente come:

Ci ha mandato dall'Africa delle notizie che sono molto preziose.

Oggi la professoressa mi ha interrogato in storia.

In quest'ultimo esempio, è necessario usare il passato prossimo perché l'azione dell'interrogare è passata, ma "oggi", cioè il tempo, non é ancora trascorso.

Esprime il prolungarsi nel tempo. Indica non la situazione, ma l'innovazione: l'ho trovato, ho capito, hanno pranzato a Curitiba, è partito, sono arrivati.

Nella frase: "Questa mattina volevo andare all'università, ma non ho potuto." L'imperfetto (volevo) esprime la situazio. ne di partenza, il passato prossimo l'avvenimento, la novità, Il fatto singolo: al contempo, l'azione è riferita al presente.

Nell'esposizione narrativa (storica), per esempio nei Promessi Sposi, il passato prossimo è estremamente raro. In questo romanzo del Manzoni, il passato prossimo s'incontra nelle parti del discorso diretto, che hanno riferimento al presente: me l'avete promesso..., che prova m'avete data?..., è uscito..., io ho capito tutto., tu m'hai inteso..., l'ho trovato..., avete sentito..., non ho fatto nulla..., ci lian dato fuoco?..., dov'è andato?..., quand'è partito?..., ho voluto venire..., piú d'uno m'ha detto.

Per questa sua particolare caratteristica di essere un tempo passato, $\mathrm{ma}$ in relazione col presente, il passato prossimo si presta ad un uso assai piú frequente, piú vivo e piú vario che non il passato remoto.

In alcune regioni settentrionali come Piemonte, Lombardia e Veneto, il passato prossimo sostituisce il passato remoto, come nel dialetto veneto: va per diese ani che'l xè morto - "son quasi dieci anni che mori", l'altra setimana go fato su sto gemo "l'altra settimana avvolsi questo gomitolo".

Questa preferenza per il passato prossimo si giustifica perché esso avvicina a noi il passato e ce lo fa rivivere come se fosse presente.

Nella narrazione il passato prossimo viene usato quando l'interesse è rivolto alla generalità d'un'azione, senza alcun riferimento a un momento definito (passato indefinito) $\mathrm{cfr}$. se il Padre degli uomini mi chiamasse a rendimento di conti, io gli dirò: non ho rapito il pane, non ho perseguitato l'infelice, non ho abbandonato l'amico... ho spartito il mio pane con lindigente (Foscolo), in opposizione al passato remoto, che concentra l'azione su un momento determinato, cfr. allora prese una sedia, la trasportò vicino al letto... s'inginocchiò 
sul tapetto... Si segnò due volte, si rialzò. Poi mise la sedia al suo posto (Serao, "Fantasia", 363).

Nella sua indefinitezza atemporale, il passato prossimo è concepibile anche in qualche dialetto dell'area meridionale estrema, in cui di norma si ha solo il passato remoto.

\section{GLI AUSILIARI}

La serie delle forme composte è infatti propria a tutte le lingue neolatine. Le forme composte esprimono il termine, quelle semplici non lo esprimono. Per conseguenza, in spagnolo he cantado esprime il termine di fronte a canto, cosi come in italiano la forma corrispondente ho cantato dovrebbe esprimere il termine di fronte al presente canto.

Il passato prossimo è un tempo composto e si forma con l'aiuto del presente dei verbi ausiliari essere o avere ognuno dei quali ha una coniugazione propria - piú il participio passato del verbo che si vuol coniugare.

I due ausiliari sono in distribuzione complementare: tutti i verbi hanno necessariamente o l'uno o l'altro - lui è arrivato, lui ha mangiato.

Essere è richiesto da una dozzina di verbi soltanto e avere da tutti gli altri.

Il verbo avere è l'ausiliare caratteristico per la forma attiva dei verbi transitivi. Il verbo essere è l'ausiliare caratteristico per la forma passiva e per la forma rifflessiva o pronominale, rispettivamente:

Il bambino è vestito dalla mamma.

Le ragazze si sono pettinate molto bene.

Mentre il toscano, e l'italiano hanno abbandonato avere col verbo riflessivo a partir dal XVII secolo, alcune zone del settentrione, soprattutto il Veneto, conservan tuttora tale costruzione cfr. il veronese s'à alzà, il veneziano el se ga negà, no se ga sparso sangue.

Diffusissimo è avere nell'Italia Meridionale cfr. il calabrese m'aju accattatu "mi son comprato", il salentino s'ia cangiata "s'era cambiata".

Per la forma attiva dei verbi intransitivi, non si possono dare norme sicure: alcuni prendono avere, altri essere, e l'insegnamento può venire solo dall'uso, dalla lettura di buoni scrittori, dalla consultazione del vocabolario. Prendono sempre l'ausiliare essere, quando sono usati nel significato intran. sitivo, quei verbi che hanno anche significato transitivo, come nei esempi:

La lezione è cominciata da un pezzo.

Il professore ha cominciato la lezione. 
Coi verbi impersonali esprimenti fenomeni metereologici l'uso oscilla tra essere e avere, con prevalenza del primo. Si dice è piovuto, è nevicato, è grandinato se si pensa all'azione in sé, mentre s'accentua la durata dell'azione si dice ha piovuto tutta la notte, ha nevicato due ore.

I dialetti seguono vie distinte: mentre Liguria, Lombardia ed Emilia preferiscono essere, in Piemonte, nel Veneto $\mathrm{e}$ in tutta l'Italia Meridionale, questi verbi vengon tutti costruiti con avere.

Oltre ai due ausiliari veri e propri, essere e avere, altri verbi possono, in alcuni casi speciali, far ufficio di ausiliari come il verbo venire, le cui voci possono sostituire quelle del verbo essere nella forma passiva: io vengo lodato, io vengo temuto, ecc.

Il verbo andare diventa ausiliare, qualche volta, per la sola terza persona singolare o plurale dei verbi transitivi, col significato di "è necessario", "deve essere": va fatto cosí, questi uccelletti vanno mangiati con la polenta.

Nell'esempio: "Vorrei avere tutti i soldi che vanno spesi per il campionato del calcio.", vanno spesi ha due valenze possibili, cioè. vengono spesi oppure devono essere spesi, mentre nella frase "Non hai idea di tutto il cibo che va sprecato.", va sprecato avrebbe il valore di viene sprecato.

Dai fatti si deduce che la locuzione perifrastica ha una determinata gamma di significati e che essa si limita al solo senso di necessità.

$\mathrm{E}$ anche il verbo stare può funzionare come ausiliare: Come sta scritto nel libro del destino.

Quanto ai considdetti verbi servili, volere, dovere, potere e sapere, quando usati in modo assoluto, cioè, senza aver alle proprie dipendenze un altro verbo, vogliono sempre l'ausiliare avere: es. ho voluto, ho dovuto, ho potuto. ho saputo. Quando invece, sono uniti a un altro verbo di modo infinito, vogliono l'ausiliare stesso che prenderebbe il verbo col quale si accompagnano: es. non ho dormito - non ho potuto dormire, non sono partito - non sono potuto partire.

Nel veneto, invece, il verbo principale non ha alcun influsso sulla forma dell'ausiliare: si usa sempre avere cfr. go dovú partir "son dovuto partire".

\section{CONCLUSIONE}

Il presente lavoro ha avuto lo scopo di offrire uno scorcio del tempo verbale passato prossimo, riprendendo le sue origini per poi risalire ai nostri giorni, tenendo anche conto di alcune variazioni dialettali. 
L'uso di tale tempo, fa parte preponderante nella comunicazione odierna, sia essa scritta che parlata.

\section{RESUMO}

Este trabalho pretende apresentar e ressaltar a importância, a origem, o uso e a formaçāo do Passado Próximo, tempo verbal fundamental para a comunicaçāo na língua italiana, por caracterizar-se como um tempo passado, mas com referència ao presente.

\section{REFERENCIAS BIBLIOGRAFICAS}

1. BATTAGLIA, Salvatore \& PERNICONE, Vincenzo. La grammatica italiana. Torino, Chiantore, 1951. $604 \mathrm{p}$.

2. LO CASCIO, Vincenzo. Struttura, funzione, valore di "andare + participio passato". Lingua e Stile. (III/3): 271.293, 1968.

3. PALAZZI, Fernando. Il governo delle parole. Milano, Principato Editore, $1962.468 \mathrm{p}$.

4. ROHLFS, Gerhard. Grammatica storica della lingua italiana e dei suoi dialetti; sintassi e formazione delle parole Torino, Giulio Einaudi Editore, 1969. v.3. 492 p.

5. SAMBUGAR, C.A. Fonti vive; grammatica e comporre. Firenze, La Nuova Italia, 1958. $439 \mathrm{p}$.

6. TEKAVVIC, Pavao. Saggio di un'analisi del sistema verbale italiano. Lingua c Stile. (V/1): 1.23, 1970. 